\title{
Surgical treatment of partial anterior cruciate ligament lesions: medium-term results
}

\author{
MASSIMO BERRUTO, LUCA GALA, PAOLO FERRUA, FRANCESCO UBOLDI, \\ FABRIZIO FERRARA, STEFANO PASQUALOTTO, BRUNO M. MARELLI
}

Gaetano Pini Orthopaedic Institute, Milan, Italy

\begin{abstract}
Purpose: this study was conducted to evaluate subjective and objective clinical outcomes of partial reconstruction of the anterior cruciate ligament (ACL) in comparison with complete ACL reconstruction.

Methods: three groups, each comprising 20 patients, were evaluated at a minimum follow-up of 12 months. The group 1 patients underwent partial ACL reconstruction, while those in group 2 and group 3 underwent complete ACL reconstruction, performed using either bone-patellar tendon-bone (BPTB) or quadrupled hamstring tendon (HT) grafts, respectively. The subjective outcome was evaluated using the Lysholm knee scale and the subjective International Knee Documentation Committee (IKDC) scoring system. A visual analog scale (VAS) was used for pain assessment and sporting activity was rated using the Tegner activity scale. Objective evaluation was performed using the IKDC objective form, KT-1000 arthrometer and KiRA triaxial accelerometer.

Results: at the follow-up evaluation, the mean subjective IKDC score was $86.1 \pm 10.3$ in group $1,85.2 \pm 11.1$ in group 2, and $82.7 \pm 7.8$ in group 3. The Lysholm score was $91.3 \pm 7.3$ in group $1,91.7 \pm 9.6$ in group 2, and $89.4 \pm 6.1$ in group 3. KT-1000 tests showed a mean side-to-side difference of $1.1 \mathrm{~mm} \pm 1.5 \mathrm{~mm}$ (range, $0-5 \mathrm{~mm}$ ) in group $1 ; 0.79 \mathrm{~mm} \pm 0.8 \mathrm{~mm}$ (range, $0-2 \mathrm{~mm}$ ) in group 2; and $1.45 \mathrm{~mm} \pm 1 \mathrm{~mm}$ (range, $0-3 \mathrm{~mm}$ ) in group 3 . The differences between groups were not statistically significant.
\end{abstract}

\section{Corresponding Author:}

Paolo Ferrua, MD

Gaetano Pini Orthopaedic Institute

Piazza Cardinale Andrea Ferrari 1, 20122 Milan, Italy

E-mail: paolo.ferrua81@gmail.com
Conclusions: both subjective and objective outcomes of partial ACL reconstruction were comparable to those of complete reconstruction, but partial reconstruction in the presence of a partial lesion of the ACL is considered by the authors to be more respectful of the native vascularization, innervation and anatomy of the ACL, conferring an advantage in terms of recovery of the complete function of the knee.

Level of evidence: Level III, retrospective comparative study.

Key Words: partial lesion, anterior cruciate ligament, reconstruction, accelerometer, selective bundle reconstruction.

\section{Introduction}

The anterior cruciate ligament (ACL) plays a critical role as a stabilizer in the knee, preventing anterior tibial translation and maintaining rotational stability. ACL lesions commonly occur during sporting activities and isolated ACL tears account for almost half of all ligament lesions in the knee. Weber, in 1836, was the first to describe the ultrastructure of the ACL, which consists of two functional bundles: anteromedial (AM) and posterolateral (PL) (1). The AM bundle mostly controls anterior translation of the tibia at more than $45^{\circ}$ degrees of flexion; the PL bundle, on the other hand, is non-isometric, more oblique and plays a fundamental role in rotational stability between $0^{\circ}$ and $30^{\circ}$ of flexion. Diagnosis of complete lesions is clinically easier than diagnosis of partial lesions. Indeed, recognizing a lesion involving only one bundle is difficult; according to the literature, such lesions account for 10-35\% of all ACL lesions, and symptomatic partial lesions account for only 5- 
$10 \%$ (2). Liljedal et al. (3), in 1965, first described a partial lesion of the ACL, but Odensten et al. (4) and Noyes et al. (5) were the first to analyze in depth the morphological characteristics of these lesions. Some authors $(6,7)$ have highlighted the fundamental biomechanical role of intact ACL bundles in partial lesions. The introduction of arthroscopic surgery and the possibility of performing a selective single-bundle reconstruction radically changed the therapeutic approach to partial lesions, making remnant-preserving reconstruction a valid treatment option. The purpose of this retrospective study was to evaluate the stability and functional outcome of a group of 20 patients who underwent an isolated reconstruction of either the AM or the PL bundle and to compare the findings in this group with those obtained in two groups of patients who underwent a reconstruction with an autologous bone-patellar tendon-bone (BPTB) or hamstring tendon (HT) graft. The hypothesis of the study was that both subjective and objective overall outcomes would be better in the partial reconstruction group compared with the complete reconstruction group, regardless of the graft selected.

\section{Methods}

A retrospective, comparative cohort study of patients affected by partial ACL lesion who had undergone either ACL partial reconstruction surgery (group 1) or a complete reconstruction with a BPTB (group 2) or autologous HT (group 3) graft between March 2008 and January 2012 was performed in our institution. All the surgical procedures were performed by the same surgeon and all the patients followed the same rehabilitation protocol.

\section{Study population}

In the group 1, the patients' mean age at the time of surgery was $32.7 \pm 10.2$ years (range, 17-55). All 20 patients (14 males and 6 females) presented an arthroscopically confirmed partial lesion of the ACL (16 of the AM bundle and 4 of the PL bundle) (Fig. 1). The grafts employed in the AM bundle reconstruction were: a doubled semitendinosus graft in 12 cases (Fig. 2 ), a 7-mm BPTB graft in two cases, a doubled graci-

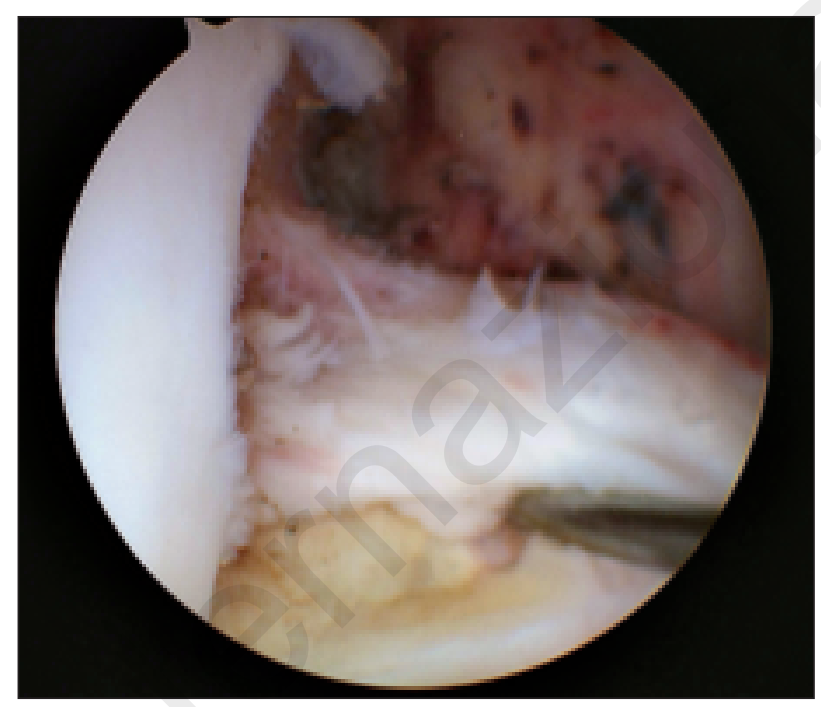

Fig. 1. Anterior cruciate ligament partial tear with healthy posterolateral bundle.

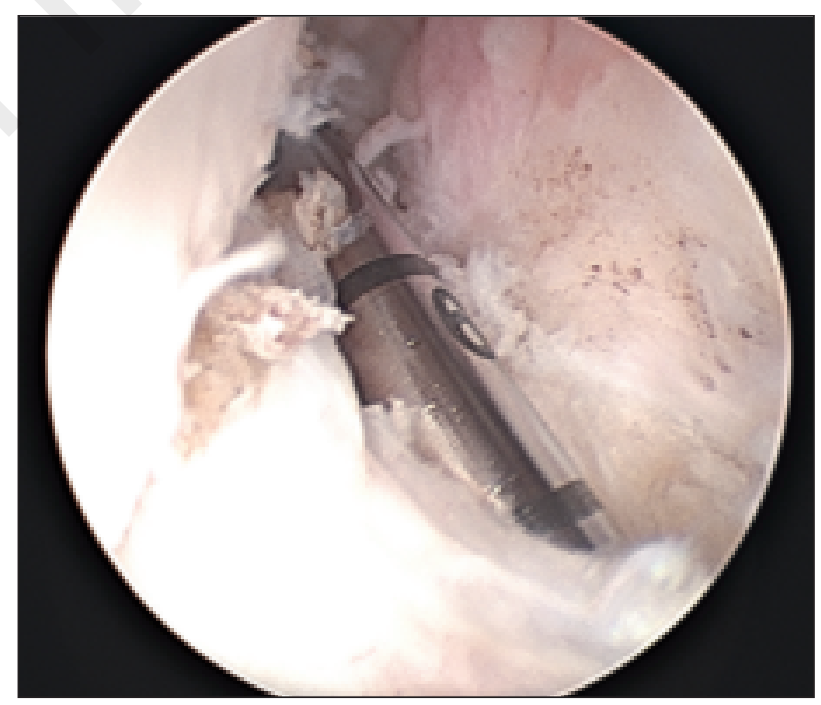

Fig. 2. Drilling of femoral tunnel using the transtibial technique.

lis graft in one case, and a doubled homologous peroneus longus graft in one case. PL bundle reconstruction was instead performed with a doubled gracilis graft (one case), doubled semitendinosus graft (two cases), or Achilles tendon allograft (one case). The graft was fixed on the tibial side using the BioIntrafix sheath and screw system (DePuy Mitek, Raynham, ME, USA) in 14 cases and a resorbable interference screw (BioRCI; Smith \& Nephew, Andover, MA, 
USA) in six patients (Fig. 3). On the femoral side, RetroButton (Arthrex, Naples, FL, USA) was used in 11 cases and Rigid-Fix (DePuy Mitek) in 8 cases; in one case a BioRCI screw was used and inserted from the anteromedial portal (AMP).

In group 2, the patients' mean age at the time of surgery was $25.6 \pm 7.5$ years $(20-43)$. In all the group 2 patients were male. The graft was fixed proximally with Rigid-Fix and distally with BioRCI screw at $30^{\circ}$ of flexion.

In group 3, the patients' mean age at the time of surgery was $32.8 \pm 8.25$ years (range, 20-47). Thirteen of these patients were male and seven were female. Femoral fixation was achieved using Rigid-Fix (DePuy Mitek) in 18 cases. In two cases the graft was fixed with a femoral Intrafix sheath and screw system (DePuy Mitek) inserted through the AMP. The graft was fixed on the tibial side with BioIntrafix in 18 cases and with BioRCI screw in two cases.

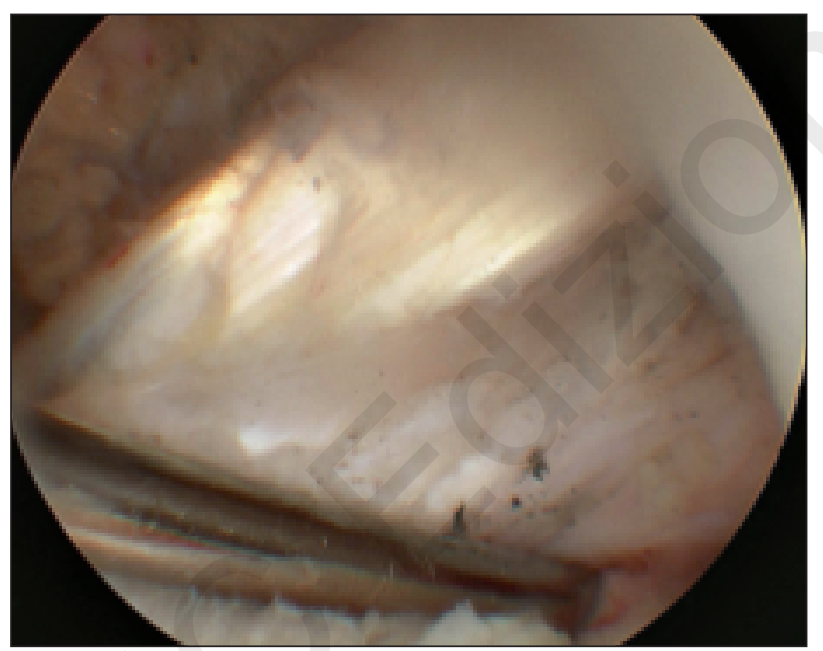

Fig. 3. Anteromedial single-bundle anterior cruciate ligament reconstruction.

\section{Outcome measurements}

Functional outcomes were evaluated subjectively and objectively by the means of the Lysholm knee scale and the International Knee Documentation Committee (IKDC) scoring system. Return to sporting activity was defined using the Tegner activity scale. Pain was assessed using a visual analog scale (VAS). Anterior tibial translation was tested with the KT-1000 arthro- meter (MED metric, San Diego, CA, USA) for objective evaluation of sagittal stability at $15 \mathrm{lb}(6.8 \mathrm{~kg})$ and $20 \mathrm{lb}(9 \mathrm{~kg})$ active displacement and with a manual maximum force (8). Only the latter measurement is presented, since it has been shown to be the strongest discriminant (9). Knee joint stability was determined in both the healthy and the injured knee and a side-toside difference $>3 \mathrm{~mm}$ was considered positive (10). The KiRA (Kinematic Rapid Assessment; OrthoKey, Lewes, DE, USA) triaxial accelerometer was used to quantify rotational stability after ACL reconstruction (11). The KiRA accelerometer was also used to record minimum, maximum and range of rotational acceleration of the tibial plateau (12).

\section{Statistical analysis}

Descriptive statistics were calculated. Comparisons of post-treatment scores in each group were calculated using the Kruskal-Wallis rank sum test. Analysis was carried out using the Microsoft Excel 2013 Analysis ToolPack (Microsoft Corporation, Redmond, WA, USA) and $\mathrm{R}$ version 3.1.0 (The R Foundation for Statistical Computing).

\section{Results}

Clinical evaluation was performed at an average follow-up of $23.8 \pm 8.3$ months (range, 12-36) in group 1, $26.7 \pm 7.3$ months (range, 16-40) in group 2, and $27.3 \pm 6.5$ months (range, 16-36) in group 3. The Lysholm knee score was $91.3 \pm 7.3$ points in group 1 , $91.7 \pm 9.6$ points in group 2 , and $89.4 \pm 6.1$ points in group 3. The mean subjective IKDC score was $86.1 \pm 10.3$ in group $1,85.2 \pm 11.1$ in group 2, and $82.7 \pm 7.8$ in group 3 . The mean Tegner score was $6 \pm 1.3$ points (range, 4-9) in group $1,6 \pm 1.7$ points (range, 3-9) in group 2 , and $5.2 \pm 1.5$ points (range, 39) in group 3. In group 1 , the mean VAS score was $0.63 \pm 1.3$ (range, $0-5$ ) and 13 patients $(65 \%)$ reported complete absence of pain (VAS $=0$ ). In group 2 , the mean VAS score was $0.37 \pm 0.6$ (range, 0 -2), with 14 patients $(70 \%)$ reporting complete absence of pain. In group 3, the mean VAS score was $0.37 \pm 0.6$ (range, 0 2), with 14 patients (70\%) found to be completely asymptomatic. In group 1, 15 patients (75\%) were 
graded A according to the IKDC objective form, four patients $(20 \%)$ were graded B, and only one patient (5\%) was graded C. In group 2, 16 patients $(80 \%)$ were graded A according to the IKDC objective form and $4(20 \%)$ were graded B. In group 3, 13 patients (65\%) were graded A according to the IKDC objective form, and 7 patients (35\%) were graded B.

KT-1000 tests showed a mean side-to-side difference of $1.05 \pm 1.5 \mathrm{~mm}$ (range, 0-5) in group 1, $0.79 \pm 0.8$ $\mathrm{mm}$ (range, $0-2$ ) in group 2, and $1.45 \pm 1 \mathrm{~mm}$ (range, $0-3)$ in group 3.

The mean values for maximum, minimum and mean acceleration, obtained using the KiRA accelerometer, were $1.9 \pm 0.2,-0.89 \pm 0.6$ and $2.8 \pm 0.4$, respectively, in group $1,1.8 \pm 0.2,-0.6 \pm 0.3$ and $2.7 \pm 0.6$, respectively, in group 2 , and $2 \pm 0.2,-0.9 \pm 0.5$ and $3 \pm 0.6$, respectively, in group 3.

No statistically significant difference either in subjective or objective outcome was found between groups (Tab. 1).

Only one failure was recorded in these three groups: a patient who received an isolated reconstruction of the PL bundle came to our attention after a new trauma (suffered while playing football), complaining of pain and subjective instability. A complete lesion of the ACL was diagnosed and confirmed with arthroscopic examination. The patient underwent revision surgery with complete reconstruction of the ACL using a soft tissue allograft.

\section{Discussion}

The most important finding of this study was that partial reconstruction of the ACL gives good results in restoring anterior and rotational stability, comparable to the subjective results obtained using gold standard procedures.

Adachi and Ochi (13) compared a group of 40 patients undergoing a reconstruction of the AM or PL bundle with another group of patients undergoing complete ACL reconstruction: the results were superior in the partial group, both in passive stability and proprioception. The same authors also published a minimum two-year follow-up study in 45 patients which showed good results in all of them (14). Siebold et al. (15) reported very good results in their preliminary study in which partial reconstructions were performed using duplicated semitendinosus grafts. Buda et al. (16) reported good or excellent results in $95.7 \%$ of cases in two prospective series of 28 and 47 patients who underwent a partial reconstruction performed using an "over the top" technique. The advantage of this technique could be that it preserves the native femoral footprint of the ACL, avoiding damaging it during drilling of the femoral tunnel. A retrospective study by Ahn et al. in 53 patients showed only one failure (17); in particular, on MRI, they found 12 "cyclops-like" lesion without any symptoms of impingement. Sonnery-Cottet et al. (18) reported the results of AM bundle reconstruction using semitendinosus grafts and which showed an excellent outcome in laxity prevention, with only two patients showing postoperative extension lags that required an arthrolysis. It was suggested that, in order to prevent this kind of complication, grafts wider than 7-8 mm should not be implanted. A multicenter study by the French Society of Arthroscopy (SFA) (19) compared two groups of patients with AM bundle lesions: one group (29 patients) underwent a partial reconstruction and the other (25 patients) a standard reconstruction. The results in the two groups were comparable, as in our

Table 1. Subjective and objective functional results.

\begin{tabular}{|c|c|c|c|c|}
\hline & Group 1 (Partial) & Group 2 (BPTB) & Group 3 (HT) & $P$ value \\
\hline Mean subjective IKDC score & $86.1 \pm 10.3$ & $85.2 \pm 11.1$ & $82.7 \pm 7.8$ & $>0.05$ \\
\hline Mean Lysholm knee score & $91.3 \pm 7.3$ & $91.7 \pm 9.6$ & $89.4 \pm 6.1$ & $>0.05$ \\
\hline Mean Tegner activity scale score & $6 \pm 1.3$ & $6 \pm 1.7$ & $5.2 \pm 1.5$ & $>0.05$ \\
\hline Mean KT-1000 side-to-side difference (mm) & $1.1 \pm 1.5$ & $0.8 \pm 0.8$ & $1.5 \pm 1$ & $>0.05$ \\
\hline Mean KiRA maximum acceleration & $1.2 \pm 0.2$ & $1.8 \pm 0.2$ & $2 \pm 0.2$ & $>0.05$ \\
\hline Mean KiRA minimum acceleration & $-0.9 \pm 0.6$ & $-0.6 \pm 0.3$ & $-0.9 \pm 0.5$ & $>0.05$ \\
\hline Mean KiRA acceleration range & $2.8 \pm 0.4$ & $2.7 \pm 0.6$ & $3 \pm 0.6$ & $>0.05$ \\
\hline
\end{tabular}


study, but proprioceptive recovery was better when preserving the PL bundle.

Partial ACL reconstruction has many advantages: the technique is more anatomical, respecting native footprints in both femoral and tibial insertion, and it exploits the vascularization and proprioceptive receptors of the intact bundle. Dodds and Arnozcky studied ACL vascularization observing a relevant contribution to the vascular supply of ACL graft by preserved ACL remnant (20). The time required for maturation and remodeling of the graft was 12 months or longer, in partial contrast to what was reported by Falconiero et al., who observed a shorter interval (6 months) (21). Buda et al. pointed out the strong correlation between a normal MRI signal of the intact bundle (25 out of 28) and good clinical results (16). When preserving the intact residual bundle in an ACL reconstruction, a portion of the proprioceptive innervation of the ligament is also retained, allowing better active stability control that could make for a faster and easier return to sporting activities, as shown by Van Eck et al. (22). Arnoczky described mechanoceptors similar to Golgi's tendon organs under the synovial membrane of the ACL and hypothesized they could have a proprioceptive function (23). Preservation of the ACL remnants has also been advocated by Adachi et al., who found a positive correlation between the number of mechanoceptors and the degree of accuracy in detecting joint position (24). Chouteau et al. found no difference in proprioception versus the healthy contralateral knee at 3.4 years of follow-up in 15 patients who underwent an AM reconstruction (25). Another possible advantage of partial reconstruction is the protective effect of the preserved bundle on the reconstructed one during the early rehabilitation, which could allow faster rehabilitation protocols. No specific complication of this technique was observed in our series or in the literature, but bulky grafts should be avoided in partial reconstructions in order to prevent postoperative anterior impingement and extension lag.

In conclusion, in this series the functional outcomes of partial reconstructions of the ACL were found to be comparable to those of standard complete reconstructions, regardless of the technique used and the graft employed (HT or BPTB). On the basis of these data, it may be argued that partial reconstruction of the
ACL does not offer any objective advantage. However, on the basis of our clinical experience and observations we consider partial reconstruction to be more anatomical, more respectful of the vascularization and proprioception, and possibly able to promote a faster recovery of coordination, functionality and efficiency of the knee joint.

We believe that, particularly in high-demanding athletes and in the presence of a functional preserved bundle, partial reconstruction of the ACL is clearly advantageous in terms of recovery of complete function of the knee. The main issues in these patients are the accuracy of the diagnosis and of the objective evaluation of the preserved bundle. A careful clinical evaluation supported by objective instruments (KT-1000 and triaxial accelerometer) is mandatory but these findings have to be confirmed under anesthesia and with a complete arthroscopic evaluation.

\section{References}

1. Wetz HH, Jacob HA. Functional anatomy and kinematics of the femorotibial joint. Results of research 1836-1950. Orthopade. 2001;30:135-144.

2. Pujol N, Colombet P, Cucurulo T, et al. Natural history of partial anterior cruciate ligament tears: a systematic literature review. Orthop Traumatol Surg Res. 2012;98(8 Suppl):S16064.

3. Liljedahl SO, Lindvall N, Wetterfors J. Early diagnosis and treatment of acute ruptures of the anterior cruciate ligament: a clinical and arthrographic study of forty-eight cases. J Bone Joint Surg Am. 1965;47:1503-1513.

4. Odensten M, Lysholm J, Gillquist J. The course of partial anterior cruciate ligament ruptures. Am J Sports Med. 1985;13:183-186.

5. Noyes FR, Mc Ginnis GH. Controversy about treatment of the knee with anterior cruciate laxity. Clin Orthop Relat Res. 1985;(198): 61-75.

6. Amis AA. The functions of the fibre bundles of the anterior cruciate ligament in anterior drawer, rotational laxity and the pivot shift. Knee Surg Sports Traumatol Arthrosc. 2012; 20:613-620.

7. Petersen W, Zantop T. Partial rupture of the anterior cruciate ligament. Arthroscopy. 2006;22:1143-1145.

8. Sernert N, Kartus J, Kohler K. Evaluation of the reproducibility of the KT-1000 arthrometer. Scand J Med Sci Sports. 2001;11:120-125.

9. Back BR Jr, Warren RF, Flynn WM, et al. Arthrometric evaluation of knees that have a torn anterior cruciate ligament. J Bone Joint Surg Am. 1990;72:1299-1306.

10. Daniel DM, Stone ML, Sachs R, et al. Instrumented measurement of anterior knee laxity in patients with acute anterior cruciate ligament disruption. Am J Sports Med. 1985; 13:401-407.

11. Berruto M, Uboldi F, Gala L, Marelli B, Albisetti W. Is triaxial accelerometer reliable in the evaluation and grading of the 
knee pivot-shift phenomenon? Knee Surg Sports Traumatol Arthrosc. 2013;21:981-985.

12. Lopomo N, Zaffagnini S, Signorelli C, et al. An original clinical methodology for non-invasive assessment of pivot shift test. Comput Methods Biomech Biomed Engin. 2012; 15: 1323-1328.

13. Adachi N, Ochi M, Uchio Y, et al. Anterior cruciate ligament augmentation under arthroscopy. A minimum 2-year followup in 40 patients. Arch Orthop Trauma Surg. 2000;120:128133.

14. Ochi M, Adachi N, Uchio Y, et al. A minimum 2-year follow up after selective anteromedial or posterolateral bundle anterior cruciate ligament reconstruction. Arthroscopy. 2009; 25:117-122.

15. Siebold R, Fu FH. Assessment and augmentation of symptomatic anteromedial or posterolateral bundle tears of the anterior cruciate ligament. Arthroscopy. 2008;24:1289-1298.

16. Buda R, Ferruzi A, Vannini F, et al. Augmentation technique with semitendinosus and gracilis tendons in chronic partial lesions of the ACL: clinical and arthrometric analysis. Knee Surg Sports Traumatol Arthrosc. 2006;14:1101-1107.

17. Ahn JH, Wang JH, Lee YS, et al. Anterior cruciate ligament reconstruction using remnant preservation and a femoral tensioning technique: clinical and magnetic resonance imaging results. Arthroscopy. 2011;27:1079-1089.

18. Sonnery-Cottet B, Lavoie F, Ogassawara R, et al. Selective anteromedial bundle reconstruction in partial ACL tears: a series of 36 patients with mean 24 months follow up. Knee Surg Sports Traumatol Arthrosc. 2010;18:47-51.

19. Pujol N, Colombet P, Potel JF, et al. Anterior cruciate ligament reconstruction in partial tear: selective anteromedial bundle reconstruction conserving the posterolateral remnant versus single-bundle anatomic ACL reconstruction: preliminary 1 -year results of a prospective randomized study. Orthop Traumatol Surg Res. 2012;98(8 Suppl):S171-177.

20. Dodds JA, Arnoczky SP. Anatomy of the anterior cruciate ligament: a blueprint for repair and reconstruction. Arthroscopy. 1994;10:132-139.

21. Falconiero RP, DiStefano VJ, Cook TM. Revascularization and ligamentization of autogenous anterior cruciate ligament grafts in humans. Arthroscopy. 1998;14:197-205.

22. Van Eck CF, Kopf S, Irrgang JJ, et al. Single-bundle versus double bundle reconstruction for anterior cruciate ligament rupture: a meta-analysis-does anatomy matter? Arthroscopy. 2012;28:405-424.

23. Arnoczky SP. Anatomy of the anterior cruciate ligament. Clin Orthop Relat Res. 1983;(172):19-25.

24. Adachi N, Ochi M, Uchio Y, et al. Mechanoreceptors in the anterior cruciate ligament contribute to the joint position sense. Acta Orthop Scand. 2002;73:330-334.

25. Chouteau J, Testa R, Viste A, et al. Knee rotational laxity and proprioceptive function 2 years after partial ACL reconstruction. Knee Surg Sports Traumatol Arthrosc. 2012; 20: 762-766. 